\title{
Rarefied Poiseuille Flow in a Circular Tube
}

\author{
Oksana Germider ${ }^{1}$ and Vasily Popov ${ }^{1, *}$ \\ ${ }^{1}$ Northern (Arctic) Federal University named after M.V. Lomonosov, RU-163002, Arkhangelsk, Russia
}

\begin{abstract}
An isothermal rarefied gas flow through a long circular tube due to longitudinal pressure gradient (a three-dimensional Poiseuille problem) was studied using the linearized Bhatnagar-Gross-Krook model kinetic equation over the whole range of the Knudsen numbers covering both free molecular and hydrodynamic regimes. The solution of the model kinetic equation with the diffuse boundary condition is obtained by the collocation method. This approach is based on the Chebyshev polynomials and rational Chebyshev functions. Choosing the zeros of Chebyshev polynomials in the multivariate range of integration for the collocation points, we reduce this problem to a set of algebraic equations. Based on the proposed approach, we have calculated the mass and the heat fluxes through the tube. The obtained results have also been compared with other studies. The developed approach may also be applied to a more general class of problems of rarefied gas flows in micro- and nanotubes.
\end{abstract}

\section{Introduction}

In a recent work [1] a newly developed version of the collocation method by the Chebyshev polynomials [2, 3] and rational Chebyshev functions [4, 5] was used to solve the diffusion equation with initial-boundary conditions over a long time domain. In this work, we develop that method for the case of rarefied gas flow in a cylindrical tube. The investigation of rarefied gas flows in micro and nanotubes is based on kinetic theory of gases. The simplest approach to an inter-atomic force law is to use Bhatnagar-Gross-Krook (BGK) model [6] where the collision process is replaced by a statistical distribution with the velocity of the scattered atom is independent of the velocity of the incident atom [7]. Under the assumption that the gas is subjected to only small perturbations, the BGK equation can be linearized and cast into the form of a linear integro-differential equation. For a circular cross section, the dimension of the problem can be reduced by passing to polar coordinates in physical space. To simplify the gas wall interaction, it is assumed that scattering is diffuse. As a result, we obtain a three-dimensional kinetic equation with homogeneous boundary condition. A solution of the homogeneous boundary value problem was constructed in [8-11]. In [9] the problem of a rarefied gas flow in a cylindrical tube was solved using a discrete-ordinates method. In [10] the same problem was solved using a classical spectral approach based on the Legendre polynomials. In the earlier works [8] and [11] results were obtained by the integro-moment method and method approximations, respectively.

To find an alternative approach for a solving of model kinetic equations in three-dimensional Poiseuille problem, which might be applied to a more general class of problems, we developed in this work a Chebyshev collocation method, which converts the threedimensional kinetic equation to a matrix equation corresponding to a system of linear algebraic equations. The aim of the present paper is to calculate based proposed approach the gas flow through a cylindrical tube over the whole range of the Knudsen number.

\section{Mathematical formulation of the problem}

Let us consider steady motion of a monoatomic rarefied gas through a long cylindrical channel with radius $R^{\prime}$. We assume that the channel length is much larger than $R^{\prime}$. A constant pressure gradient is assumed to be maintained along the channel axis. As a boundary condition at the channel wall, we will use the Maxwell model of diffuse reflection. We will consider the flow of gas in the vicinity of the middle of the channel. To do this, we introduce a Cartesian coordinate system Oxyz, whose $\mathrm{Oz}$ axis is assumed to be directed along the channel axis. As the size scale, we will use the radius of the cylinder. It is assumed that the presser change is small as follows:

$$
G_{p}=\frac{1}{p_{0}} \frac{d p}{d z}, \quad\left|G_{p}\right| \square 1 .
$$

Here $p_{0}$ is the pressure at the point that is selected as the origin, $z=z^{\prime} / R^{\prime}$. Taking into account (1), the state of a rarefied gas at the point $(\mathbf{r}, \mathbf{C})$ we will determined by the distribution function $f(\mathbf{r}, \mathbf{C})$ of gas molecules by coordinates and velocities 


$$
\begin{aligned}
f(\mathbf{r}, \mathbf{C}) & =f_{0}(C)(1+h(\mathbf{r}, \mathbf{C})), \\
h(\mathbf{r}, \mathbf{C}) & =G_{p}\left(z+C_{z} Z^{\prime}\left(\rho, C_{\perp}, \psi\right)\right) .
\end{aligned}
$$

Here $f_{0}(C)=n_{0}(\beta / \pi)^{3 / 2} \exp \left(-C^{2}\right), \mathbf{r}=\mathbf{r}^{\prime} / R^{\prime}$ is the dimensionless radius vector of gas molecules, $\mathbf{C}=\beta^{1 / 2} \mathbf{v}$ is the dimensionless molecular velocity, $\beta=m /\left(2 k_{B} T_{0}\right)$, $m$ is the mass of gas molecules, $k_{B}$ is the Boltzmann constant, $T_{0}$ and $n_{0}$ are the temperature and the concentration of gas molecules at the point that is selected as the origin and $h(\mathbf{r}, \mathbf{C})$ is the perturbation function. In a configuration space and a space of velocities, we will use the cylindrical coordinates $(\rho, \varphi, z): \quad x=\rho \cos \varphi, \quad y=\rho \sin \varphi, \quad z ; \quad\left(C_{\perp}, \psi, C_{z}\right):$ $C_{\rho}=C_{\perp} \cos \psi, C_{\varphi}=C_{\perp} \sin \psi, C_{z}$.

In order to find the distribution of gas molecules by coordinates and velocities, we make use of the linearized BGK kinetic model of Boltzmann equation, which in the cylindrical coordinate system has the form $[4,9]$

$$
\begin{gathered}
\left(\frac{\partial Z^{\prime}}{\partial \rho} \cos \psi-\frac{\partial Z^{\prime}}{\partial \psi} \frac{\sin \psi}{\rho}\right) C_{\perp}+\mathrm{Kn}^{-1} Z^{\prime}\left(\rho, C_{\perp}, \psi\right)+1= \\
\quad=\frac{1}{\pi \mathrm{Kn}} \int_{0}^{+\infty} \int_{0}^{2 \pi} \exp \left(-C^{\prime 2}{ }_{\perp}\right) C_{\perp} Z^{\prime}\left(\rho, C^{\prime}{ }_{\perp}, \psi^{\prime}\right) d C^{\prime}{ }_{\perp} d \psi^{\prime},(2)
\end{gathered}
$$

where $\mathrm{Kn}=l_{g} / R^{\prime}$ is the Knudsen number, $l_{g}=\eta_{g} \beta^{-1 / 2} / p$ is the mean free path and $\eta_{g}$ is dynamic viscosity of the gas. Entering the notation $\zeta=\cos \psi$, we rewrite the equation (2) for finding the function $Z^{\prime}\left(\rho, C_{\perp}, \psi\right)=Z\left(\rho, C_{\perp}, \zeta\right)$ in the form

$$
\begin{aligned}
& \left(\frac{\partial Z}{\partial \rho} \zeta+\frac{\partial Z}{\partial \zeta} \frac{\left(1-\zeta^{2}\right)}{\rho}\right) C_{\perp}+\mathrm{Kn}^{-1} Z\left(\rho, C_{\perp}, \zeta\right)+1= \\
= & \frac{2}{\operatorname{Kn} \pi} \int_{0}^{+\infty} C_{\perp}^{\prime} \exp \left(-C_{\perp}^{\prime 2}\right) \int_{-1}^{1} \frac{Z\left(\rho, C_{\perp}^{\prime}, \zeta^{\prime}\right)}{\sqrt{1-\zeta^{\prime 2}}} d \zeta^{\prime} d C_{\perp}^{\prime} .
\end{aligned}
$$

The boundary conditions for $Z\left(\rho, C_{\perp}, \zeta\right)$ is specified using the diffuse reflection model [11]

$$
Z\left(1, C_{\perp}, \zeta\right)=0, \quad \zeta<0 .
$$

Taking into account the statistical meaning of the distribution function, we find the dimensionless $z$ components of the mass flow rate and the heat flow vector in the duct $[12,13]$

$$
\begin{gathered}
U_{z}(\rho)=\frac{G_{p}}{\pi} \int_{0}^{+\infty} \exp \left(-C_{\perp}^{2}\right) C_{\perp} \int_{-1}^{1} \frac{Z\left(\rho, C_{\perp}, \zeta\right)}{\sqrt{1-\zeta^{2}}} d \zeta d C_{\perp}, \quad(5) \\
q_{z}(\rho)=\frac{G_{p}}{\pi} \int_{0}^{+\infty} \exp \left(-C_{\perp}^{2}\right) C_{\perp}^{3} \int_{-1}^{1} \frac{Z\left(\rho, C_{\perp}, \zeta\right)}{\sqrt{1-\zeta^{2}}} d \zeta d C_{\perp}-U_{z}(\rho) .
\end{gathered}
$$

Respectively the dimensionless mass and heat fluxes through the chancel cross section are determined according to [14]

$$
J_{M}=4 \int_{0}^{1} U_{z}(\rho) \rho d \rho, \quad J_{Q}=4 \int_{0}^{1} q_{z}(\rho) \rho d \rho .
$$

To find the numerical solution of the boundary problem (3) and (4) we use the Chebyshev polynomials $[2,3]$ and the rational Chebyshev functions $[4,5]$.

\section{Solution to the problem}

The function $Z\left(\rho, C_{\perp}, \zeta\right)$ defined on $[0,1] \times[-1,1] \times[0, \infty)$ may be expanded in the Chebyshev polynomials $\left\{T_{j_{1}}\left(\rho_{*}\right)\right\}, \quad\left\{T_{j_{2}}(\zeta)\right\}$ and the rational Chebyshev functions $\left\{R_{j_{3}}\left(C_{\perp}\right)\right\}$ as

$$
Z\left(\rho, C_{\perp}, \zeta\right)=\sum_{\mathbf{j}=\mathbf{0}}^{\infty} a_{\mathbf{j}} T_{j_{1}}\left(\rho_{*}\right) T_{j_{2}}(\zeta) R_{j_{3}}\left(C_{\perp}\right)
$$

where $a_{\mathbf{j}}$ are unknown coefficients and $\mathbf{j}=\left(j_{1}, j_{2}, j_{3}\right)$,

$$
\rho_{*}=2 \rho-1, \quad C_{\perp, *}=\frac{C_{\perp}-1}{C_{\perp}+1}, \quad R_{j_{3}}\left(C_{\perp}\right)=T_{j_{3}}\left(C_{\perp, *}\right)
$$

To calculate the Chebyshev polynomials, we used the following recurrence relations [2]

$$
\begin{gathered}
T_{0}(x)=1, \quad T_{1}(x)=x, \\
T_{j}(x)=2 x T_{j-1}(x)-T_{j-2}(x), \quad j \geq 2 .
\end{gathered}
$$

Let the approximation of $Z\left(\rho, C_{\perp}, \zeta\right)$ be obtained by truncating the series (7) as

$$
Z\left(\rho, C_{\perp}, \zeta\right)=\mathbf{T}_{1}\left(\rho_{*}\right) \mathbf{Q}(\zeta) \mathbf{W}\left(C_{\perp, *}\right) \mathbf{A},
$$

where $\mathbf{n}=\left(n_{1}, n_{2}, n_{3}\right), n_{k}^{\prime}=n_{k}+1(k=\overline{1,3})$,

$$
\mathbf{T}_{1}\left(\rho_{*}\right)=\mathbf{T}_{1 \times n_{1}^{\prime}}\left(\rho_{*}\right), \quad \mathbf{T}_{1 \times n}(x)=\left(T_{0}(x) T_{1}(x) \ldots T_{n}(x)\right),
$$

$\mathbf{Q}(\zeta)$ and $\mathbf{W}\left(C_{\perp, *}\right)$ are block matrices

$$
\begin{gathered}
\mathbf{Q}(\zeta)=\boldsymbol{\Phi}_{n_{1}^{\prime} \times n_{1}^{\prime} n_{2}^{\prime}}(\zeta), \quad \mathbf{W}\left(C_{\perp, *}\right)=\mathbf{\Phi}_{n_{1}^{\prime} n_{2}^{\prime} \times n_{1}^{\prime} n_{2}^{\prime} n_{3}^{\prime}}\left(C_{\perp, *}\right), \\
\boldsymbol{\Phi}_{m \times m n}(x)=\operatorname{diag}\left\{\mathbf{T}_{1 \times n}(x), \mathbf{T}_{1 \times n}(x), \ldots \mathbf{T}_{1 \times n}(x)\right\},
\end{gathered}
$$

and $\mathbf{A}$ is the $n_{1}^{\prime} n_{2}^{\prime} n_{3}^{\prime} \times 1$ matrix as

$$
\mathbf{A}=\left(a_{000} a_{001} \ldots a_{00 n_{3}} a_{010} \ldots a_{n_{1} n_{2}, n_{3}-1} a_{n_{1} n_{2} n_{3}}\right)^{T}
$$

The operational matrices of the first derivatives of matrices $\mathbf{T}_{1}(\hat{\rho})$ and $\mathbf{T}_{2}(\zeta)$, respectively, of a variable $\rho$ and $\zeta$ may be defined by 


$$
\frac{d \mathbf{T}_{1}\left(\rho_{*}\right)}{d \rho}=2 \mathbf{T}_{1}\left(\rho_{*}\right) \mathbf{J}_{1}, \quad \frac{d \mathbf{T}_{2}(\zeta)}{d \zeta}=\mathbf{T}_{2}(\zeta) \mathbf{J}_{2},
$$

where $\mathbf{J}_{\mathbf{k}}=\mathbf{J}_{n_{k}^{\prime} \times n_{k}^{\prime}}$ is the square operational matrix ( $k=1,2$ ), whose nonzero elements are given explicitly by [14]

$$
\left(J_{k}\right)_{i, j+1}=\left\{\begin{array}{lllr}
j, & i=1, & j & \text { odd, } \\
2 j & j>i-1, & i, j & \text { even, } \\
2 j & j>i-1, i>1, & i, j & \text { odd. }
\end{array}\right.
$$

Taking into account the orthogonality of the polynomials $T_{j_{2}}(\zeta)$, we obtain

$$
\int_{-1}^{1} \frac{T_{j_{2}}(\zeta)}{\sqrt{1-\zeta^{2}}}= \begin{cases}\pi, & j_{2}=0, \\ 0, & j_{2} \neq 0 .\end{cases}
$$

Expressing from (8) the variables $\rho$ and $C_{\perp}$ in terms of $\rho_{*}$ and $C_{\perp, *}$ and taking into account (9)-(11), we write the equation (3) as

$$
\begin{gathered}
\Lambda\left(\rho_{*}, \zeta, C_{\perp, *}\right) \mathbf{A}=-1, \\
\Lambda\left(\rho_{*}, \zeta, C_{\perp, *}\right)=\left(\zeta \mathbf{T}_{1}\left(\rho_{*}\right) \mathbf{J}_{1} \mathbf{Q}(\zeta)+\frac{\left(1-\zeta^{2}\right)}{\hat{\rho}+1} \mathbf{T}_{1}\left(\rho_{*}\right) \mathbf{Q}^{\prime}(\zeta)\right) \times \\
\times \frac{2\left(C_{\perp, *}+1\right)}{1-C_{\perp, *}} \mathbf{W}\left(C_{\perp, *}\right)+\mathrm{Kn}^{-1} \mathbf{T}_{1}\left(\rho_{*}\right) \mathbf{Q}(\zeta) \mathbf{W}\left(C_{\perp, *}\right)- \\
-4 \mathrm{Kn}^{-1} \mathbf{P}\left(\rho_{*}\right),
\end{gathered}
$$

where $\quad \mathbf{Q}_{n_{1}^{\prime} \times n_{1}^{\prime} n_{2}^{\prime}}^{\prime}(\zeta)=\operatorname{diag}\left\{\mathbf{T}_{2}(\zeta) \mathbf{J}_{2}, \mathbf{T}_{2}(\zeta) \mathbf{J}_{2}, \ldots \mathbf{T}_{2}(\zeta) \mathbf{J}_{2}\right\}$ and $\mathbf{P}$ is the $1 \times n_{1}^{\prime} n_{2}^{\prime} n_{3}^{\prime}$ matrix, whose nonzero elements are given explicitly by

$$
\begin{gathered}
P_{1, n_{2}^{\prime} n_{3_{1} i_{1}+i_{3}+1}}\left(\rho_{*}\right)=\left(T_{1}\right)_{1, i_{1}+1}\left(\rho_{*}\right) I_{i_{3}}, \quad i_{1,3}=0,1, \ldots, n_{1,3}, \\
2 I_{i_{3}}=\int_{0}^{+\infty} \exp \left(-C_{\perp}^{2}\right) C_{\perp} R_{i_{3}}\left(C_{\perp}\right) d C_{\perp} .
\end{gathered}
$$

Now, our aim is to calculate the values of $I_{i_{3}}$ defined by (14). Replacing the variable $C_{\perp}$ with $\hat{C}_{\perp}$ and using (8), we write the integral $I_{i_{3}}$ as

$$
\begin{gathered}
I_{i_{3}}=\int_{-1}^{1} w_{i_{3}}\left(C_{\perp, *}\right) d C_{\perp, *}, \quad i_{3}=0,1, \ldots, n_{3}, \\
w_{i_{3}}\left(C_{\perp, *}\right)=\frac{\exp \left(-\frac{\left(1+C_{\perp, *}\right)^{2}}{\left(1-C_{\perp, *}\right)^{2}}\right)\left(1+C_{\perp, *}\right) T_{i_{3}}\left(C_{\perp, *}\right)}{\left(1-C_{\perp, *}\right)^{3}} .
\end{gathered}
$$

The function $w_{i_{3}}\left(C_{\perp, *}\right)$ defined on $[-1,1]$ can be expanded in the Chebyshev polynomials as

$$
w_{i_{3}}\left(C_{\perp, *}\right)=\sum_{i=0}^{n} " b_{i} T_{i}\left(C_{\perp, *}\right),
$$

where $\sum "$ is the finite summation with first and last terms halved.

Substituting the expression (15) into (13), we assume that $n$ is an even number. Taking into account relation

$$
\int_{-1}^{1} T_{l}(x) d x=\left\{\begin{array}{cl}
\frac{2}{1-l^{2}}, & \text { l even }, \\
0, & l \text { odd },
\end{array}\right.
$$

we obtain

$$
I_{i_{3}, n}=2 \sum_{l=0}^{n / 2} " \frac{b_{2 l}}{1-4 l^{2}}=b_{0}-\frac{2}{3} b_{2}-\frac{2}{15} b_{4}-\ldots-\frac{1}{n^{2}-1} b_{n} .
$$

Unknown coefficients $b_{2 l}(l=\overline{0, n / 2})$ in (17) we will find, taking into account that [2]

$$
\sum_{i=0}^{n} " T_{i}\left(C_{\perp, *, k}\right) T_{j}\left(C_{\perp, *, k}\right)= \begin{cases}n, & (i=j=0) \vee(i=j=n), \\ 0, & 0 \leq i \neq j \leq n, \\ \frac{n}{2}, & 0<i=j<n .\end{cases}
$$

Here, $C_{\perp, *, k}(k=\overline{0, n})$ are the extrema of the polynomial $T_{n}\left(C_{\perp, *}\right) \quad$ on the interval $C_{\perp, *} \in[-1 ; 1]$ defined as

$$
C_{\perp, *, k}=\cos \left(\frac{\pi(n-k)}{n}\right) .
$$

Multiplying the left and right parts of the equation (15) by $T_{j}\left(\hat{C}_{\perp}\right)(j=\overline{0, n})$, we substitute the points (19) into the these equations. By using (18), we have

$$
b_{j}=\frac{2}{n} \sum_{k=0}^{n} " w_{i_{3}}\left(C_{\perp, *, k}\right) T_{j}\left(C_{\perp, *, k}\right), \quad j=\overline{0, n} .
$$

By substituting (20) into (17), we obtain

$$
\begin{gathered}
I_{i_{3}, n}=\frac{4}{n} \sum_{k=0}^{n} " \tau_{e x}\left(C_{\perp, *, k}, n\right) w_{i_{3}}\left(C_{\perp, *, k}\right), \\
\tau_{e x}\left(C_{\perp,,, k}, n\right)=\sum_{i=0}^{n / 2} " \frac{T_{i}\left(C_{\perp, *, k}\right)}{1-4 i^{2}} .
\end{gathered}
$$

For the proposed method, the error assessment is presented [14] as

$$
E_{i_{3}, n}^{*}=\left|I_{i_{3}, n}-I_{i_{3}, n / 2}\right| .
$$

Note that the $T_{n / 2}$ have the extrema $C_{\perp,{ }^{*}, r}^{\prime}$ equal the extrema $C_{\perp, *, 2 r}$ of $T_{n}$, so that $C_{\perp, *, r}=C_{\perp, *, 2 r}$ $(r=\overline{0, n / 2})$. Indeed, using (19), we have 


$$
C_{\perp, *, r}^{\prime}=\cos \left(\frac{\pi(n / 2-r)}{n / 2}\right)=\cos \left(\frac{\pi(n-2 r)}{n}\right) .
$$

This implies that an automatic quadrature routine that doubles the number of nodes can reuse the calculated values of the function $w_{i_{3}}$. In Table 1 the values of error estimates of $E_{i_{3}, n}^{*}$ with $n=40$ for $i_{3}=\overline{0, n_{3}}$ and $n_{3}=10$ are listed. The comparison between the values of $I_{i_{3}, 40}$ calculated by the formula (21) and $I_{i_{3}, G}$ obtained by Gauss method in Maple software with the Digits environment variable assigned to be 10 is provided in the Table 2 as $E_{i_{3}, n}=\left|I_{i_{3}, n}-I_{i_{3}, G}\right|$. should be used.

Table 1. Values of $I_{i_{3}, 40}$ and error estimates.

\begin{tabular}{|c|c|c|c|c|c|c|}
\hline$i_{3}$ & 0 & 1 & 2 & 4 & 9 & 10 \\
\hline$E_{i_{3}, 40}^{*}$ & $1 \cdot 10^{-6}$ & $3 \cdot 10^{-7}$ & $3 \cdot 10^{-6}$ & $3 \cdot 10^{-6}$ & $1 \cdot 10^{-5}$ & $2 \cdot 10^{-5}$ \\
\hline$E_{i_{3}, 40}$ & $2 \cdot 10^{-9}$ & $3 \cdot 10^{-11}$ & $1 \cdot 10^{-10}$ & $3 \cdot 10^{-10}$ & $3 \cdot 10^{-10}$ & $4 \cdot 10^{-10}$ \\
\hline
\end{tabular}

The value 40 of $n$ we will use to calculate the elements of the matrix $\mathbf{P}$ by (13) and (21). Now, we consider the problem (3) and (4). Utilizing boundary condition (4) as following:

$$
\mathbf{\Omega}\left(1, \zeta, C_{\perp, *}\right) \mathbf{A}=0, \quad \zeta<0,
$$

where $\mathbf{\Omega}\left(1, \zeta, C_{\perp, *}\right)=\mathbf{T}_{\mathbf{1}}(1) \mathbf{Q}(\zeta) \mathbf{W}\left(C_{\perp, *}\right)$.

As the collocation points in (12) and (23), we will use the zeros for $x$ in $[-1,1]$ of the polynomial $T_{n_{i}^{\prime}}(x)[2]$

$$
x_{i, k}=\cos \left(\frac{\pi\left(2 n_{i}-2 k+1\right)}{2\left(n_{i}+1\right)}\right) \text {, }
$$

where $x_{1, k}=\rho_{*, k}, \quad x_{2, k}=\zeta_{k}$ and $x_{3, k}=C_{\perp, *, k} \quad\left(k=\overline{0, n_{i}}\right.$, $i=\overline{1,3}$ ).

Substituting (24) into (12), we arrive at a system of linear $n_{1}^{\prime} n_{2}^{\prime} n_{3}^{\prime}$-equations, in which we replace equations with $\rho_{*}=\rho_{*, n_{1}}, \zeta=\zeta_{k_{2}}$ and $C_{\perp}=C_{\perp, *, k_{3}}$ to the equations (24) with the values of the variables $\rho_{*}=1, \zeta=\zeta_{k_{2}}$ and $C_{\perp, *}=C_{\perp, *, k_{3}}\left(k_{2}=\overline{0, k_{2}^{\prime}}, \quad k_{3}=\overline{0, n_{3}}\right)$. Here $k_{2}^{\prime}$ is an index such that $\zeta_{k_{2}^{\prime}}<0$ and $\zeta_{k_{2}^{\prime}+1} \geq 0$. Then,

$$
\mathbf{B A}=\mathbf{F},
$$

where $\mathbf{B}$ and $\mathbf{F}$ are matrices with sizes $n_{1}^{\prime} n_{2}^{\prime} n_{3}^{\prime} \times n_{1}^{\prime} n_{2}^{\prime} n_{3}^{\prime}$ and $n_{1}^{\prime} n_{2}^{\prime} n_{3}^{\prime} \times 1$, respectively:

$$
\mathbf{B}=\left(\begin{array}{c}
\boldsymbol{\Lambda}\left(\rho_{*, 0}, \zeta_{0}, C_{\perp, *, 0}\right) \\
\boldsymbol{\Lambda}\left(\rho_{*, 0}, \zeta_{0}, C_{\perp, *, 1}\right) \\
\ldots \\
\boldsymbol{\Lambda}\left(\rho_{*, n_{1}-1}, \zeta_{n_{2}}, C_{\perp, *, n_{3}}\right) \\
\boldsymbol{\Omega}\left(1, \zeta_{0}, C_{\perp, *, 0}\right) \\
\ldots \\
\boldsymbol{\Omega}\left(1, \zeta_{k_{2}^{\prime}}, C_{\perp, *, n_{3}}\right) \\
\boldsymbol{\Lambda}\left(\rho_{*, n_{1}}, \zeta_{k_{2}^{\prime}+1}, C_{\perp, *, 0}\right) \\
\ldots \\
\boldsymbol{\Lambda}\left(\rho_{*, n_{1}}, \zeta_{n_{2}}, C_{\perp, *, n_{3}-1}\right) \\
\boldsymbol{\Lambda}\left(\rho_{*, n_{1}}, \zeta_{n_{2}}, C_{\perp, *, n_{3}}\right)
\end{array}\right), \mathbf{F}=\left(\begin{array}{c}
-1 \\
-1 \\
\ldots \\
-1 \\
0 \\
0 \\
-1 \\
\ldots \\
-1 \\
-1
\end{array}\right) .
$$

The algebraic system (25) is solved by the LU method in Maple software.

Now, we find the macroscopic parameters of the gas in the duct. Substituting (7) into (5) and taking into account (13), we obtain

$$
\begin{gathered}
U_{z}(\rho)=2 G_{p} \mathbf{P}\left(\rho_{*}\right) \mathbf{A}, \\
q_{z}(\rho)=2 G_{p} \mathbf{P}^{*}\left(\rho_{*}\right) \mathbf{A}-U_{z}(\rho) .
\end{gathered}
$$

Here, $\mathbf{P}^{*}$ is the $1 \times n_{1}^{\prime} n_{2}^{\prime} n_{3}^{\prime}$ matrix, in which the elements different from zero are determined by the elements of the matrix $\mathbf{T}_{1}(\hat{\rho})$ and the integral $I_{i_{3}}^{*}$ as

$$
\begin{gathered}
P_{1, n_{2}^{\prime} n_{3}^{\prime} i_{1}+i_{3}+1}^{*}\left(\rho_{*}\right)=\left(T_{1}\right)_{1, i_{1}+1}\left(\rho_{*}\right) I_{i_{3}}^{*}, \quad i_{1,3}=0,1, \ldots, n_{1,3}, \\
2 I_{i_{3}}^{*}=\int_{0}^{+\infty} \exp \left(-C_{\perp}^{2}\right) C_{\perp}^{3} R_{i_{3}}\left(C_{\perp}\right) d C_{\perp} .
\end{gathered}
$$

Note that the integral $I_{i_{3}}^{*}$ can be calculate in the same way as $I_{i_{3}}$ :

$$
\begin{gathered}
I_{i_{3}, n}^{*}=\frac{4}{n} \sum_{k=0}^{n} " \tau_{e x}\left(C_{\perp, *, k}, n\right) w_{i_{3}}^{*}\left(C_{\perp, *, k}\right), \\
w_{i_{3}}^{*}\left(C_{\perp, *}\right)=\frac{w_{i_{3}}\left(C_{\perp, *}\right)\left(1+C_{\perp, *}\right)^{2}}{\left(1-C_{\perp, *}\right)^{2}} .
\end{gathered}
$$

From (26) and (27) and properties of the Chebyshev polynomials (16) and

$$
2 T_{j}(x) T_{k}(x)=T_{j+k}(x)+T_{|j-k|}(x),
$$

the expressions (6) for dimensionless mass and heat fluxes become

$$
J_{M}=2 G_{p} \int_{-1}^{1} \mathbf{P}\left(\rho_{*}\right)\left(1+T_{1}\left(\rho_{*}\right)\right) d \rho_{*} \mathbf{A}=
$$

$$
\begin{gathered}
=4 G_{p} \sum_{i_{3}=0}^{n_{3}} I_{i_{3}}\left(\sum_{i_{1}=0}^{n_{1} / 2} \frac{a_{2 n_{2}^{\prime} n_{3}^{\prime} i_{1}+i_{3}+1,1}}{1-4 i_{1}^{2}}+\sum_{i_{1}=0}^{n_{1} / 2-1} \frac{a_{n_{2}^{\prime} n_{3}^{\prime}\left(2 i_{1}+1\right)+i_{3}+1,1}}{4-\left(2 i_{1}+1\right)^{2}}\right), \\
J_{Q}=2 G_{p} \int_{-1}^{1} \mathbf{P}^{*}\left(\rho_{*}\right)\left(1+T_{1}\left(\rho_{*}\right)\right) d \rho_{*} \mathbf{A}-J_{M}=-J_{M}+
\end{gathered}
$$




$$
+4 G_{p} \sum_{i_{3}=0}^{n_{3}} I_{i_{3}}^{*}\left(\sum_{i_{1}=0}^{n_{1} / 2} \frac{a_{2 n_{2}^{\prime} n_{3}^{\prime} i_{1}+i_{3}+1,1}}{1-4 i_{1}^{2}}+\sum_{i_{1}=0}^{n_{1} / 2-1} \frac{a_{n_{2}^{\prime} n_{3}^{\prime}\left(2 i_{1}+1\right)+i_{3}+1,1}}{4-\left(2 i_{1}+1\right)^{2}}\right),
$$

where $n_{1}$ is even.

To estimate the error assessment for the mass and heat flux by (28) and (29) we use the formula [14, 15]

$$
E_{\mathbf{n}}^{* *}=\left|J_{\mathbf{n}}-J_{\mathbf{n} / 2}\right| \text {. }
$$

\section{Results and Discussion}

The values of the mass and the heat fluxes calculated by (28) and (29) are presented in the Tables 2 and 3 for $n_{1}=2 n_{2,3}=n^{*}$ and $2 n_{1,3}=n_{2}=n^{* *}$. Analogous results for this problem of the Poiseuille flow obtained in [9] and [18] by the discrete coordinate and velocities method (Table 4). As can be seen from the Tables 2-4, the results obtained the proposed method in good agreement with the analogous results from [9] and [18]. Note that the use of the Chebyshev polynomials and rational Chebyshev functions in proposed paper allowed to reduce the calculations thus accelerating the convergence. Thus, in [18] the solution was constructed using a finite difference scheme of the first order on a non-uniform $1000 \times 1000$ grid in physical space.

Table 2. The mass flux $-J_{M} / G_{p}$ for various values of $\mathrm{Kn}$.

\begin{tabular}{|c|c|c|c|c|c|}
\hline $\mathrm{Kn}^{-1}$ & $n^{*}=10$ & $n^{*}=20$ & $\mathrm{Kn}^{-1}$ & $n^{* *}=10$ & $n^{* *}=20$ \\
\hline 0.5 & 1.3815 & 1.3869 & 0.1 & 1.4270 & 1.4043 \\
\hline 1.0 & 1.4569 & 1.4586 & 0.2 & 1.3808 & 1.3817 \\
\hline 2.0 & 1.6537 & 1.6581 & & & \\
\hline 5.0 & 2.3497 & 2.3493 & & & \\
\hline 10.0 & 3.2879 & 3.5657 & & & \\
\hline
\end{tabular}

Table 3. The mass flux $J_{Q} / G_{p}$ for various values of $\mathrm{Kn}$.

\begin{tabular}{|c|c|c|c|c|c|}
\hline $\mathrm{Kn}^{-1}$ & $n^{*}=10$ & $n^{*}=20$ & $\mathrm{Kn}^{-1}$ & $n^{* *}=10$ & $n^{* *}=20$ \\
\hline 0.5 & 0.4167 & 0.4172 & 0.1 & 0.5846 & 0.5968 \\
\hline 1.0 & 0.3211 & 0.3218 & 0.2 & 0.5303 & 0.5292 \\
\hline 2.0 & 0.2267 & 0.2272 & & & \\
\hline 5.0 & 0.1219 & 0.1222 & & & \\
\hline 10.0 & 0.0638 & 0.0686 & & & \\
\hline
\end{tabular}

\section{Conclusion}

In this paper, by applying the Chebyshev polynomials and rational Chebyshev functions for the three-variable function, we solved the BGK equation with diffuse boundary conditions. Thus, after the use of the set of collocation points, the required numerical solution is found to be equivalent to the solution of a linear system of algebraic equations written in matrix form. The values of the mass and the heat fluxes through the channel cross section have been calculated. The resultant expressions are analyzed numerically. From the conducted comparison, it turns out that the proposed method converges quickly and can be used to solve the BGK equation in the Poiseuille flow problem in a long channel with a more complex cross section configuration.

Table 4. The mass and heat fluxes in $[9,18]$.

\begin{tabular}{|c|c|c|c|}
\hline \multirow{2}{*}{$\mathrm{Kn}^{-1}$} & \multicolumn{2}{|c|}{$-J_{M} / G_{p}$} & $J_{Q} / G_{p}$ \\
\cline { 2 - 4 } & {$[7,8]$} & {$[16]$} & {$[7,8]$} \\
\hline 0.1 & 1.40396 & 1.403 & 0.597479 \\
\hline 0.2 & 1.38159 & 1.381 & 0.529371 \\
\hline 0.5 & 1.38665 & 1.386 & 0.417068 \\
\hline 1.0 & 1.45829 & 1.458 & 0.321726 \\
\hline 2.0 & 1.65765 & 1.657 & 0.227118 \\
\hline 5.0 & 2.34833 & 2.348 & 0.122228 \\
\hline 10.0 & 3.56412 & 3.563 & 0.068581 \\
\hline
\end{tabular}

\section{References}

1. A. Baseri, S. Abbasbandy, E. Babolian, Appl. Mathematics and Computation 322, 55-65 (2018)

2. J. Mason, D. Handscomb, Chebyshev Polynomials (CRC Press, Florida, 2003)

3. M.M. Khader, N.H. Sweilam, Applied Mathematical Modelling, 37 (24), 9819-9828 (2013).

4. J. Boyd, Chebyshev and Fourier spectral methods (DOVER Publications, Mineola, 2000).

5. H.N. Soloklo, M.M. Farsangi, Scientia Iranica 20(3), 771-777 (2013).

6. M.M.R. Williams, Z. angew. Math. Phys. 52, 500516 (2001).

7. C. Cercignani, Mathematical Methods in Kinetic Theory (Plenum Press, New York, 1990).

8. S. Lo, S. Loyalka, Z. Angew. Math. Phys. 33, 419424 (1982).

9. C. Siewert, Journal of Computational Physics 160, 470-480 (2000).

10. L. Barichello, C. Kamphorst, P. Rodrigues, Journal of Pure and Applied Mathematics 51, 181-187 (2009).

11. D. Valougeorgis, J. Thomas, Phys. Fluids 29, 423429 (1986).

12. M. Kogan, Rarefied Gas Dynamics (Plenum, New York, 1969).

13. F. Sharipov, V. Seleznev, Journal of Physical and Chemical Reference Data 27, 657-706 (1998).

14. E. Tohidi, Ain Shams Engineering Journal 6, 373379 (2015).

15. J. Berntsen, T. Espelid, A. Genz, ACM TOMS 17, 437-451 (1991).

16. A. Genz, R. Kass, Journal of Computational and Graphical Statistics 6, 92-111 (1997).

17. E.Di Napoli, M.A. Hermanns, H. Iliev, A. Lintermann, A. Peyser, High-Performance Scientific Computing (Springer, Cham, 2017).

18. I. Graur, F. Sharipov, European Journal of Mechanics B/Fluids 27, 335-345 (2008). 